\title{
IEEE 802.11ac wireless delivery of 4kUHD video: The impact of packet loss
}

\author{
Anthony Adeyemi-Ejeye ${ }^{1}$, Mohammed Alreshoodi ${ }^{2}$, Laith Al-Jobouri ${ }^{3}$, Martin Fleury ${ }^{3}$, John Woods ${ }^{3}$, Mujtaba Medhi ${ }^{1}$ \\ ${ }^{1}$ Kingston Univ., London, U.K., ${ }^{2}$ Qassim Univ., Buraydah, Saudi Arabia, ${ }^{3}$ Univ. of Essex, Colchester, U.K. \\ \{a.adeyemi-ejeye,m.mehdi\}@kingston.ac.uk; mo.alreshoodi@qu.edu.sa; \{lamoha,fleum,woodjt\}@essex.ac.uk
}

\begin{abstract}
This paper examines the 4kUHD video quality from streaming over an IEEE 802.11ac wireless channel, given measured levels of packet loss. Findings suggest that there is a strong content dependency to loss impact upon video quality but that, for short-range transmission, the quality is acceptable, making 4kUHD feasible on head-mounted displays.
\end{abstract}

Keywords-4kUHD; head-mounted display; IEEE 802.11ac; Packet Loss Visibility; video streaming

\section{INTRODUCTION}

The authors of [1] report a low-power High Efficiency Video Coding (HEVC) codec processor that is capable of delivering 4kUHD (Ultra High Definition) video $(3840 \times$ 2160 p pixels/frame, $16 \times 9$ aspect ratio) at $30 \mathrm{fps}$ to Long Term Evolution (LTE) and 5G portable devices. Portable devices are potentially better at displaying 4kUHD than traditional $\mathrm{TV}$ screens because 4kUHD is best viewed close-up. Alternatively, a $3 \mathrm{~m}$ viewing distance would require an 80 " screen. Though smart-phone 4kUHD displays already exist, even then users may find it hard to distinguish $1080 \mathrm{p}$ resolution from 4kUHD. However, head-mounted displays (HMD's) for (say) Augmented Reality (AR) can benefit from this resolution because the display is very close to the eyes.

In this paper, we consider another aspect to $4 \mathrm{kUHD}$, which is the feasibility of streaming over 'lossy' wireless channels. Of potential wireless technologies, IEEE 802.11ac [2] provides a high-throughput wireless LAN in the unlicensed $5 \mathrm{GHz}$ band. In theory, a single spatial channel has a maximum throughput of 867 Mbps. This is principally due to an increase in mandatory channel width to $80 \mathrm{MHz}$ and up to 256-Quadrature Amplitude Modulation (QAM). However, when 256-QAM is selected, the impact of noise increases significantly. Moreover, the increased channel width that restricts $802.11 \mathrm{ac}$ operation to the $5 \mathrm{GHz}$ band also results in shorter wavelengths at this frequency, affecting the propagation behavior. Thus, unless some form of HTTP Adaptive Streaming (HAS) is employed, leading to larger buffers and a significant risk of display stalls [3], packet loss visibility (PLV) (video quality after UDP-based streaming) becomes a concern for high-resolution video.

Our experiments firstly considered what levels of packet loss would occur in a number of indoor transmission scenarios and then determined the viewing quality after HEVC compression. Prior study by others has considered either the likely IEEE 802.11ac data-rates (Wave 1) in an indoor environment, as in [4], or the subjective video quality after error-free delivery, as in [5]. However, few researchers appear to have examined PLV within 4kUHD video, linking this to packet loss rates for an indoor environment. Since Wave 1, Wave 2 IEEE 802.11ac has introduced Multi-User (MU)Multiple Input Multiple Output (MIMO) antennas $(2 \times 2$ configuration), allowing high datarate streaming even when several AR devices are connected to the same access point (or for 3D display). As there are already commercial HD HMD's, including wireless $\mathrm{HD}$, with applications such as personal digital cinema and gaming, the feasibility of streaming 4kUHD to HMD's should be explored.

\section{METHODOLOGY}

Four 4kUHD adaptations of well-known test sequences were employed. Table I records the characteristics of the sequences in terms of recommendation ITU-T P.910's Spatial Index (SI) and Temporal Index (TI). A Graphics Processing Unit (GPU)-assisted encoder was implemented based around enhancing the H.264/Advanced Video Coding (AVC) implementation of [6]. However, the encoding time was reduced to $5 \mathrm{~s}$ (rather than $7 \mathrm{~s}$ for [6]) for 500 frames of 4kUHD Sintel, with similar reductions for the other test sequences. This reduction was achieved by zero-copy memory transfer to the GPU rather than the memcpy mechanism in [6]. Streaming was at a Constant Bitrate (CBR) of $20 \mathrm{Mbps}$ with the H.264/AVC High profile, with Group of Pictures (GoP) size 40 and frame structure IPPP... The aim was to assess packet loss rates (PLRs) in a typical indoor environment (average of 10 trials for each data point). The configuration of IEEE 802.11ac was similar to [4], using the high-throughput features present in the Broadcom BCM4360 chipset, as given in Table II.

\section{FINDINGS}

Fig. 1 selects the packet losses at $10 \mathrm{~m}$ and $20 \mathrm{~m}$ within an office (mean of 20 tests). For the $10 \mathrm{~m}$ transmission there were no obstructions but for the $20 \mathrm{~m}$ experiment typical office furniture was present. It was also found that, when it was necessary to pass through intervening partition walls, there was a rapid fall off of available datarates. From Fig. 1, it may appear that up to $20 \mathrm{~m}$ PLRs are not distinguished by distance. However, the PLRs for higher-activity Sintel in particular are noticeably higher.

TABLE I Test video sequences content type

\begin{tabular}{llll}
\multicolumn{3}{c}{ TABLE I } & Test video sequences content type \\
\hline Video sequence & SI & TI & Motion classification \\
\hline Coast & 10.84 & 16.92 & Moderate \\
News & 17.52 & 21.24 & Moderate \\
Foreman & 19.71 & 38.29 & High \\
Sintel & 16.39 & 72.26 & High \\
\hline
\end{tabular}


$\underline{\text { TABLE II Settings for IEEE 802.11ac measurements }}$

\begin{tabular}{ll}
\hline \multicolumn{1}{c}{ Setting } & \multicolumn{1}{c}{ Value } \\
\hline Channel bandwidth & $80 \mathrm{MHz}$ \\
Channel number & $36(5.180 \mathrm{GHz})$ \\
No. of spatial channels & $3(3 \times 3 \mathrm{MIMO})$ \\
Transport protocol & $\mathrm{UDP}$ \\
Datagram size & $1472 \mathrm{~B}$ \\
Modulation & $256-\mathrm{QAM}$ \\
\hline
\end{tabular}

Inspection of the output frame rates of the hardwareassisted encoder, Fig. 2, showed that the coding complexity reduced Sintel's output to $15 \mathrm{fps}$, whereas Coast's output approached $20 \mathrm{fps}$, with the other two clip's rate between that in order of motion activity (Table I). We, therefore, surmise that the total time during which a video was transmitted exposed the video to more packet loss events, despite the fact that channel 36 (Table II) was chosen to avoid external interference and transmission was at night. Nonetheless, Fig. 1 appears to show likely PLRs, which might be reduced for less active sequences to about $0.1 \%$ with application-layer channel coding.

Video quality in Fig. 3 was assessed with the well-known Structural Similarity (SSIM) index which more than PSNR reflects the response of the Human Visual System but approaches PSNR's computation speed. To bring the results more in line with contemporary developments, the HEVC codec, FFmpeg implementation, was configured in its Main profile, to output at a CBR of $13.5 \mathrm{Mbps}$ (approximately equivalent in quality to an H.264/AVC rate of $20 \mathrm{Mbps}$ ). The GoP size was 25 with an IBBPBBP... frame structure at 25 fps. From Fig. 3's plots, particularly for PLRs between $0.2 \%$ and $0.5 \%$, it is clear that motion activity is a strong determinant of the packet loss impact. Coast in particular benefits from its lower activity. Certainly, one would not want to stream $4 \mathrm{kUHD}$ video in this configuration if the PLR was above $0.5 \%$. However, Fig. 1 implies that a PLR above $0.5 \%$ may not often occur. SSIM does not assess temporal quality and a frame rate of $60 \mathrm{fps}$ rather than $25 \mathrm{fps}$ may eventually be required.

\section{CONCLUSION}

Wireless IEEE 802.11ac seems a likely way to transmit compressed 4kUHD. Provided the transmission range is not

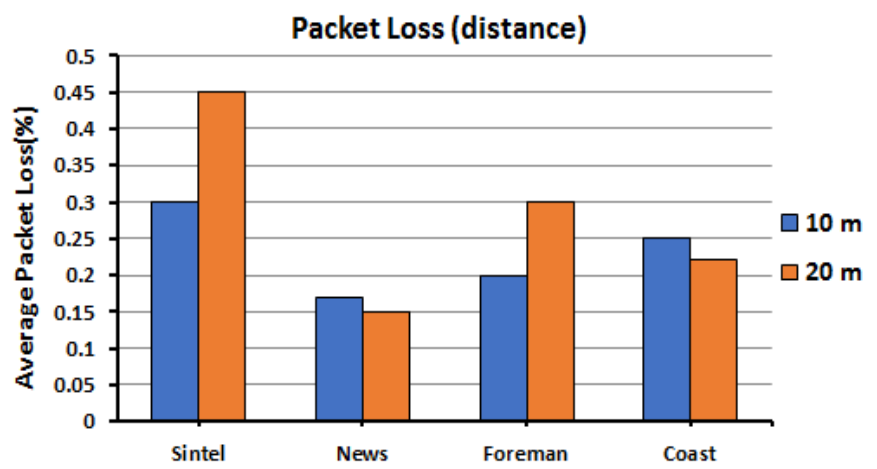

Fig. 1. Packet loss at different distances during IEEE 802.11ac transmission of $4 \mathrm{kUHD}$ video

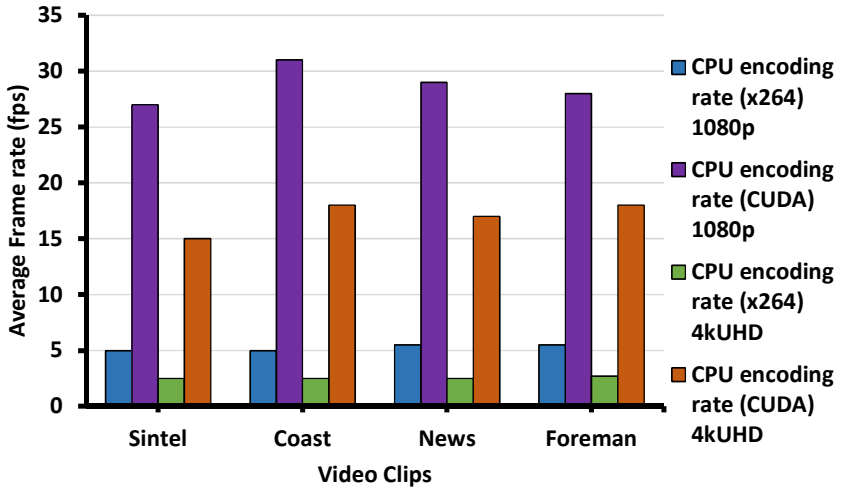

Fig. 2. Comparison of achievable framerates for HD and 4kUHD video

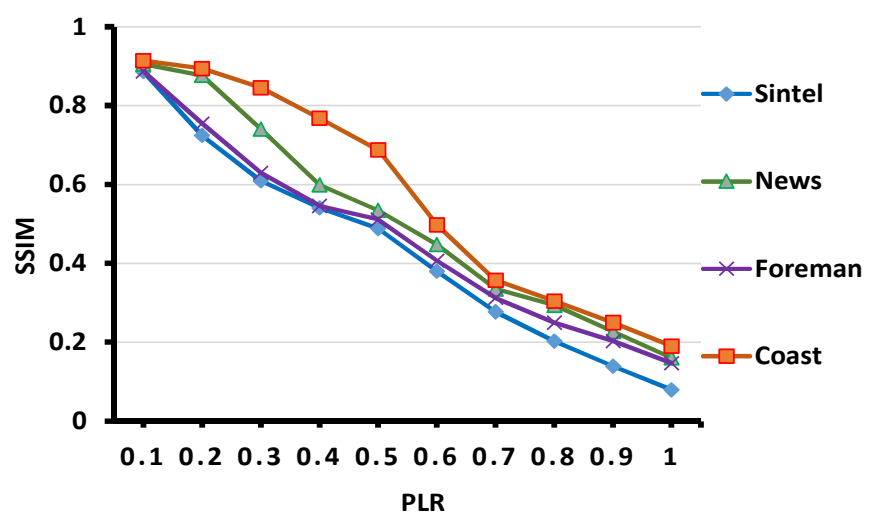

Fig. 3. SSIM video quality assessment for a range of PLR percentages with HEVC codec encoding and 4kUHD resolution

much greater than $10 \mathrm{~m}$, PLRs of less than $0.2 \%$ may be acceptable. However, high motion activity within a video, e.g. a TI over 30 in Table I, will strongly influence the perceived quality. As it is easy to classify video by such activity, contentselective streaming may be possible. However, what FEC overhead will be needed requires further investigation before a real-time HMD application such as AR is achieved.

\section{REFERENCES}

[1] S. Lee, H. Kim, and N. Eum, "Reduced complexity single-core based HEVC video codec processor for mobile 4k-UHD applications," IEEE Int. Conf. Consumer Electron., Berlin 2016.

[2] O. Bejarano and E.W. Knightly, "IEEE 802.11ac: From channelization to multi-user MIMO," IEEE Commun., vol. 51, no. 10, pp. 84-90, 2013

[3] M Seufert, S. Egger, H. Slanina, T. Zinner,T. Hoßfeld, and P. Tran-Gia, "A survey on Quality of Experience of HTTP adaptive streaming," IEEE Commun. Surveys \& Tutorials, vol. 17, no. 1, pp. 469-492, 2015.

[4] M.-D. Dianu, J. Riihijärvi, and M. Petrova, "Measurement-based study of the performance of IEEE 802.11ac in an indoor environment," in Proc. of IEEE Int.Conf. Commun., 2014.

[5] S.-H. Bae, J. Kim, M. Kim, S. Cho, and J.S. Choi, "Assessments of subjective video quality on HEVC-encoded 4K-UHD video for BeyondHDTV broadcasting services," IEEE Trans. Broadcast., vol. 59, no. 2, pp. 209-221, 2013.

[6] N. Wu, M. Wen, H. Su, J. Ren and C. Zhang, "A parallel H.264 encoder with CUDA: Mapping and evaluation," in Proc. of the IEEE 18th Int. Conf. on Parallel and Distrib. Syst., 2012, pp. 276-283. 